\title{
Metabolic syndrome severity score: range and associations with cardiovascular risk factors
}

\author{
Borislav D. Dimitrov ${ }^{1}$, Karamfil M. Bahchevanov², Penka A. Atanassova², Mitko D. Mitkov³, \\ Radka I. Massaldjieva ${ }^{4}$, Kostadin A. Chompalov², Georgi K. Hadzhipetrov²
}

\author{
1Primary Care and Population Sciences, Faculty of Medicine, University \\ of Southampton, Southampton, UK \\ ${ }^{2}$ Department of Neurology, Medical Faculty, Medical University of Plovdiv, Plovdiv, \\ Bulgaria \\ ${ }^{3}$ Department of Endocrinology, Medical Faculty, Medical University of Plovdiv, Plovdiv, \\ Bulgaria \\ ${ }^{4}$ Faculty of Public Health, Medical University of Plovdiv, Plovdiv, Bulgaria
}

Submitted: 21 August 2016

Accepted: 22 August 2016

Arch Med Sci Atheroscler Dis 2016; 1: e90-e97

DOI: 10.5114/amsad.2016.62137

Copyright @ 2016 Termedia \& Banach

\section{Abstract}

Introduction: Metabolic Syndrome Severity Score (MSSS) is a new clinical prediction rule (CPR) for diagnostic and therapeutic decisions and employs available components (sex, age, race, systolic blood pressure, waistline circumference, high-density lipoprotein, triglycerides and fasting blood glucose). The aim of our work was to perform cross-sectional pilot trial on middle-aged healthy volunteers and patients with metabolic syndrome (MetS) with and without type 2 diabetes mellitus (T2DM) for studying feasibility and implementation of MSSS and its associations with cardiovascular risk factors.

Material and methods: We approached 64 eligible participants from Bulgaria. The MSSS values, together with demographic, anthropometric, medical history, laboratory findings, CVD risk factors, QRISK2 score for 10-year cardiovascular risk and predicted heart age, were analysed. Descriptive statistics with tests for comparison (e.g., $t$-test, $\chi^{2}$ ) between groups as well as ANOVA and logistic regression were applied.

Results: We analysed data from 56 participants (aged $50.11 \pm 3.43$ years). The MSSS was higher in MetS patients (including 6 T2DM patients) than in controls $(n=29 ; 51.8 \%)$ presented as percentiles $(69.97 \%$ and $34.41 \%$, respectively) and $z$-scores $(0.60$ and -0.45 , respectively) $(p<0.05)$. The logistic regression model of MSSS indicated a positive association with MetS/T2DM cases (correctness $>85 \%, p<0.01$ ). For further validation purposes, positive correlations of MSSS with CVD risk factor as diastolic blood pressure (Rho $=0.399 ; p<0.003)$ and QRISK2 score (Rho $=0.524 ; p<0.001)$ or predicted heart age (Rho $=0.368 ; p<0.007)$ were also found.

Conclusions: The pilot study of MSSS in Bulgaria indicated feasibility and consistency of its implementation among patients with metabolic syndrome and/or T2DM and healthy volunteers.

Key words: metabolic syndrome, risk, vascular risk factors.

\section{Introduction}

The metabolic syndrome (MetS) represents a complex disease entity of mutually related pathological symptoms, signs and risk factors (dysglycaemia, dyslipidaemia, arterial hypertension and obesity of visceral

\author{
Corresponding author: \\ Borislav D. Dimitrov \\ Primary Care and \\ Population Sciences \\ Faculty of Medicine \\ University of Southampton \\ Southampton General \\ Hospital \\ Level C \\ Tremona Road \\ SO166YD Southampton, UK \\ Phone: +44 2381206138 \\ Fax: +44 2381206529 \\ E-mail: b.dimitrov@soton. \\ ac.uk
}


type) for development of type 2 diabetes mellitus (T2DM) and cardiovascular diseases (CVD) [1]. The classification of MetS in two clinical groups depending on the presence or absence of T2DM additionally widens the problem in consideration of the large variability of microvascular pathologies such as retinopathy, neuropathy and nephropathy $[2,3]$. This concept of MetS as a two-facet entity (ICD-10-CM Code E88.81 Metabolic syndrome) appears to be a very important clinical tool for the assessment of the risk of CVD and T2DM. According to a number of longitudinal studies, when MetS is present, the CVD risk is increased two-fold [4], while the T2DM risk is increased up to 4-fold [5].

The increasing incidence and prevalence of MetS worldwide ( $>20 \%$ among the adult population) [6] determine its major health, social and economic importance. Defining better the severity of MetS might represent a core aspect of a successful clinical approach for estimation of the risk of T2DM [5] and CVD [4]. The frequency of MetS in Bulgaria is $26.8 \%$ if defined by only 3 components (CVD risk factors) and further increases if defined by 4 such possible components (by 6\%) [7]. Another study has reported a prevalence of about $30.8 \%$ of MetS [8].

The diagnostic (reference) criteria for MetS are a combination of results from routine clinical and laboratory tests - systolic blood pressure, waist circumference, high-density lipoproteins (HDL) cholesterol, triglycerides and fasting blood glucose levels, according to the International Diabetes Federation (IDF). They are closely related to other parallel metabolic states and processes such as insulin resistance, oxidative stress and chronic inflammation, which, on their own, may also be considered as predictors of CVD (e.g., hsCRP) [9, 10]. However, some of the imperfections of the above diagnostic criteria lie in their binary nature (presence/absence) which does not allow for an exact estimation of the risk when marginal values are considered. Such borderline values may carry a hidden cardio-metabolic risk, as we had shown earlier for marginally increased homocysteine levels (still within the "normally" accepted range) and increased but "hidden" risk of ischaemic stroke in younger patients [11]. Similarly, we have identified new cut-off points based on predicted risk of outcome events that were below or above the previously accepted normal (reference) values of the predictors $[12,13]$ - in many cases such cut-offs are very useful in practice and may be easily incorporated into diagnostic or treatment algorithms. Unfortunately, in the case of the MetS components, such binary nature allows neither a diagnosis of MetS (when the diagnostic criteria are increased, or even borderline, but still below the accepted normal reference values) nor an adequate estimation of the severity of the underlying pathological processes. To solve this duality some authors have suggested in such situations the use of a continuous range of values $[14,15]$ which form a scale of severity of MetS. Many authors have proposed different methods for calculation of such continuous scales in adults $[16,17]$ as well as in children and teenagers [18-20]. The authors used different statistical methods to derive such scales as principal component analysis [16, 20-22] and the method of percentiles [23]. For our current study we applied the most recently suggested scale, called the metabolic syndrome severity score (MSSS), which can be calculated as a $z$-score as well as in percentiles. The score is based on both clinical and anthropometric characteristics.

The aim of our study was to perform a pilot application of MSSS to analyse its feasibility, range and associations with CVD risk factors among both patients with MetS and/or T2DM and healthy volunteers in Bulgaria.

\section{Material and methods}

\section{Participant selections}

We approached 64 consecutive volunteers from the Clinic of Endocrinology and Clinic of Neurology, Medical University Hospital "St George", Plovdiv, who were seen during the period from October 2014 to October 2015. The study was approved by the Ethical Committee of the Medical Faculty. The inclusion criteria were: age $45-55$ years, with normal daily living. The exclusion criteria were: history of cardiovascular or cerebrovascular accidents (e.g., heart attack, TIA, stroke), traumatic, degenerative or inflammatory diseases of the nervous system; epilepsy, other endocrine disorders (except T2DM) or medications which could have had metabolic effects.

\section{Clinical and laboratory investigations}

The participants who agreed to participate and provided written informed consent (according to the Declaration of Helsinki guidelines) were subjected to further interview and physical (including anthropometric), neurological and laboratory investigations. Laboratory tests: blood tests by Coulter STKS (USA); tests for glucose, urea, creatinine, total and HDL cholesterol, triglycerides and liver enzymes (Konelab 60i, Finland). According to the questionnaire on disease history, CVD risk factors and laboratory tests the volunteers were divided into two groups: 32 cases of patients with the diagnosis of metabolic syndrome (MetS, at least 3 components present according to current IDF criteria) and 32 controls without MetS (al- 
though with 1 or 2 CVD risk factors). Out of $32 \mathrm{ca}-$ ses with MetS, 6 cases had T2DM.

\section{Clinical prediction rules (CPRs)}

The clinical scales, or rules, for evaluation of CVD risk and associated pathologies and events were calculated using free online calculators. The 10-year CVD risk was assessed by the QRISK2 score, provided by the University of Nottingham, UK [24] at http://www.qrisk.org/. The predicted heart/vascular age was calculated by using data on the lipid profile according to the equation from the Framingham study in the USA [25] (Cardiovascular Disease (10-year risk) - interactive risk score calculator using lipids at www.framinghamheartstudy.org/risk-functions/cardiovascular-disease/10-year-risk.php\#). These estimates helped us to evaluate the potential risk of future CVD events for all participants.

Metabolic syndrome severity score (MSSS): MSSS calculation was performed with the online tool "METS Severity Calculator" at http://publichealth.hsc.wvu.edu/biostatistics/metabolic-syndrome-severity-calculator/mets-severity-calculator/ [20]. The equations for calculation of MSSS are based on the NHANES study in the USA with the following arguments: age, race, gender, waist circumference, triglycerides (TGL), high-density lipoprotein $(\mathrm{HDL})$ cholesterol, low-density lipoprotein (LDL) cholesterol, systolic blood pressure (SBP) and blood glucose levels [20]. The MSSS was calculated in two variants: (i) $z$-score (from minus to plus infinity) with zero (0) indicating the average severity, and (ii) percentiles (0-100\%), which can be interpreted as the growth percentiles in children.

\section{Statistical analysis}

This study was performed as a cross-sectional, case-control proof-of-concept trial to establish the prevalence of MetS, T2DM, CVD risk factors and range of MSSS and other CPRs and association within a pilot sample of the Bulgarian population. Since there were only 6 patients with T2DM, these were initially described as a separate sub-group, but they were later combined with those having only MetS to form the two main outcome populations in our study: MetS/T2DM patients (cases) and healthy volunteers (controls). Given the pilot nature of the study, no a priori power and sample size calculations were performed, but a convenience sample of consecutive eligible participants was used.

Data were analysed by descriptive statistics presented as frequency (percent) for the categorical variables and mean with standard deviation and $95 \% \mathrm{Cl}$ for the continuous variables, as appropriate. Normality of distributions of the continu- ous variables was tested by the Shapiro-Wilk test. The comparisons were made by parametric and non-parametric methods ( $t$-test, Mann-Whitney test) as well as analysis of variance (ANOVA) or $\chi^{2}$ and Fisher's exact test for categorical data. Associations were tested by odds ratio (OR) and its 95\% Cl and parametric (Pearson) or non-parametric (Spearman) correlations, as appropriate. Univariable or multivariable logistic regression analyses were applied to predict outcome as based on MSSS as adjusted for one or more possible confounders. $P$-value $<0.05$ was considered statistically significant. All analyses were performed by the statistical software packages SPSS and SAS.

\section{Results}

\section{Patients' characteristics}

The main demographic, medical history, clinical and laboratory features of all patients and controls included in the analyses are summarised in Table I. From all approached 64 eligible participants, 8 participants dropped out (5 patients and 3 controls) as they did not provide informed consent (Figure 1). Therefore, 56 participants were included in the analyses in two outcome groups: 29 (51.8\%) healthy volunteers aged $50.59 \pm 3.41$ years ( 6 men, 20.7\%; 23 women, 79.3\%) and 27 (48.2\%) patients with MetS/T2DM aged $49.59 \pm 3.43$ years (13 men, 48.1\%; 14 women, $51.9 \%)$ including 6 patients with T2DM. The frequency was $48.2 \%(95 \% \mathrm{Cl}$ : 35.1-61.3). No differences were found except for gender, with a predominating proportion of female participants among controls.

\section{Associations of MSSS with MetS/T2DM}

All risk factors and components of MetS were different, understandably, between the cases and controls $(p<0.05)$. As mentioned earlier, 6 (22.2\%) of the patients with MetS also had T2DM. Also, the additionally estimated 10 -year CVD risk was almost double in cases (7.90\%) compared to controls (3.79\%); the predicted heart age was also higher in the cases ( 65.08 years) than in controls; all differences at $p<0.01$.

In particular, the newly developed rule MSSS also had higher, more risky mean values among the cases $(p<0.01)$ in its both variants ( $z$-score and percentiles), i.e., 0.60 and $69.97 \%$, respectively (Table I). This first important validating pattern is very well illustrated in Figure 2, where it also very well seen that the significant overall difference in MSSS z-score between the two groups (Figure $2 \mathrm{~A}$ ) is due not only to the difference between healthy controls $(n=29)$ and T2DM patients $(n=6)$, but also to the significant difference compared to the patients with MetS alone $(n=21)$ (Figure $2 \mathrm{~B}$, $\left.p_{\text {ANOVA }}<0.001\right)$. 
Table I. Main socio-demographic, medical history and clinical characteristics

\begin{tabular}{|c|c|c|c|}
\hline Characteristics [units] ${ }^{*}$ & $\begin{array}{l}\text { Healthy volunteers } \\
\text { (controls) }\end{array}$ & $\begin{array}{l}\text { Patients with MetS } \\
\text { and/or T2DM (cases)* }\end{array}$ & Total \\
\hline Number (\%) & $29(51.8)$ & $27(48.2)$ & $56(100)$ \\
\hline Gender $(M / F)^{\#}$ & $6(20.7) / 23(79.3)$ & $13(48.1) / 14(51.9)$ & $19(33.9) / 37(66.1)$ \\
\hline Age & $50.59 \pm 3.41$ & $49.59 \pm 3.44$ & $50.11 \pm 3.43$ \\
\hline Education (secondary/higher) & $5(17.2) / 24(82.8)$ & $8(29.6) / 19(70.4)$ & $13(23.2) / 43(76.8)$ \\
\hline Smoking (no/ex-smoker/yes) & $13(46.4) / 7(25.0) / 8(28.6)$ & $11(20.4) / 7(13) / 8(14.8)$ & $24(24.4) / 14(25.9) / 16(29.6)$ \\
\hline Medications (no/yes) & $6(75) / 2(25)$ & $3(25) / 9(75)$ & $9(45) / 11(55)$ \\
\hline Family history (no/yes)\# & $4(44) / 5(55.6)$ & $0(0) / 12(100)$ & $4(19) / 17(81.0)$ \\
\hline $\begin{array}{l}\text { Family predisposition (mother/ } \\
\text { father) }\end{array}$ & $5(100) / 0(0)$ & $9(75) / 3(25)$ & $14(82.4) / 12(70.6)$ \\
\hline \multicolumn{4}{|c|}{ Diseases - main vascular risk factors: } \\
\hline $\begin{array}{l}\text { Cardiovascular disease (CVD) } \\
\text { (no/yes)\# }\end{array}$ & $20(71.4) / 8(28.6)$ & $11(42.3) / 15(57.7)$ & $32(57.4) / 23(42.6)$ \\
\hline $\begin{array}{l}\text { Type } 2 \text { diabetes mellitus } \\
\text { (T2DM) (no/yes) }\end{array}$ & $28(100) / 0(0)$ & $20(76.9) / 6(23.1)$ & $48(88.9) / 6(11.1)$ \\
\hline Dyslipidaemia (DLP) (no/yes) ${ }^{\#}$ & $22(81.5) / 5(18.5)$ & $14(53.8) / 12(46.2)$ & $36(67.9) / 17(32.1)$ \\
\hline $\begin{array}{l}\text { Peripheral vascular disease } \\
\text { (PVD) (no/yes) }\end{array}$ & $25(89.3) / 3(10.7)$ & $24(92.3) / 2(7.7)$ & $49(90.7) / 5(9.3)$ \\
\hline Hypertension (HT) (no/yes) & $28(100) / 0(0)$ & $10(40) / 15(60)$ & $37(71.7) / 15(28.3)$ \\
\hline Other diseases (no/yes) & $17(81) / 4(19)$ & $13(72.2) / 5(27.8)$ & $30(76.9) / 9(23.1)$ \\
\hline \multicolumn{4}{|c|}{ Components of the metabolic syndrome: } \\
\hline $\begin{array}{l}\mathrm{HDL}<1 \mathrm{mmol} / / \text { (men) } \\
\text { or } \mathrm{HDL}<1.29 \mathrm{mmol} / \mathrm{l} \text { (women) } \\
\text { or on treatment (no/yes) }\end{array}$ & $25(86.2) / 4(13.8)$ & $10(37) / 17(63.0)$ & $35(62.5) / 21(37.5)$ \\
\hline $\begin{array}{l}\mathrm{TGL}>1.7 \mathrm{mmol} / \mathrm{l} \text { and/ } \\
\text { or on treatment (no/yes) }\end{array}$ & $28(96.6) / 1(3.4)$ & $7(25.9) / 20(74.1)$ & $35(62.5) / 21(37.5)$ \\
\hline $\begin{array}{l}\text { Blood glucose }>5.6 \mathrm{mmol} / \mathrm{l} \\
\text { and/or on treatment/T2DM } \\
\text { (no/yes) }^{\mathrm{s}}\end{array}$ & $19(65.5) / 10(34.5)$ & $8(29.6) / 19(70.4)$ & $27(48.2) / 29(51.8)$ \\
\hline $\begin{array}{l}\mathrm{SBP}>130 \text { or } \mathrm{DBP}>85 \\
\text { and/or on } \\
\text { anti-hypertensive therapy } \\
\text { (no/yes) } \text { ) }^{\text {s* }}\end{array}$ & $28(96.6) / 1(3.4)$ & $11(40.7) / 16(59.3)$ & $39(69.6) / 17(30.4)$ \\
\hline $\begin{array}{l}\text { Waist circumference } \\
>94 \mathrm{~cm} \text { (men) or }>80 \mathrm{~cm} \\
\text { (women) (no/yes) }{ }^{\#}\end{array}$ & $11(37.9) / 18(62.1)$ & $3(11.1) / 24(88.9)$ & $14(25) / 42(75)$ \\
\hline Predicted heart age [years] $]^{\text {* }}$ & $52.14 \pm 9.43$ & $65.08 \pm 12.83$ & $58.37 \pm 12.86$ \\
\hline $\begin{array}{l}\text { QRISK index (10-year CVD risk } \\
\text { indicator) }[\%]^{]^{*}}\end{array}$ & $3.79 \pm 2.41$ & $7.90 \pm 7.38$ & $5.78 \pm 5.75$ \\
\hline $\begin{array}{l}\text { Metabolic syndrome severity } \\
\text { score (MSSS) }(z \text {-score })^{\varsigma *}\end{array}$ & $-0.45 \pm 0.51$ & $0.60 \pm 0.57$ & $0.059 \pm 0.76$ \\
\hline $\begin{array}{l}\text { Metabolic syndrome severity } \\
\text { score (MSSS) [percentiles] }\end{array}$ & $34.41 \pm 16.62$ & $69.97 \pm 17.13$ & $51.56 \pm 24.51$ \\
\hline
\end{tabular}

*MetS/T2DM - metabolic syndrome/type 2 diabetes mellitus; number (percent) or mean (standard deviation); the category "Yes" means the presence of the characteristics or symptom or positive (pathological) results from a diagnostic test/procedure; ${ }^{\#} p<0.05$ or ${ }^{5} p<$ 0.01 indicates significant difference between cases and controls as based on $\chi^{2}$ test or Fisher's exact test; the quantitative variables are compared by the non-parametric Mann-Whitney test; the predicted heart age (years) is calculated according to the models from the Framingham study in the USA (see text for more details); the index of 10-year cardiovascular risk (QRISK) is calculated according to the model equation provided by the University of Nottingham in England (see text for more details). 


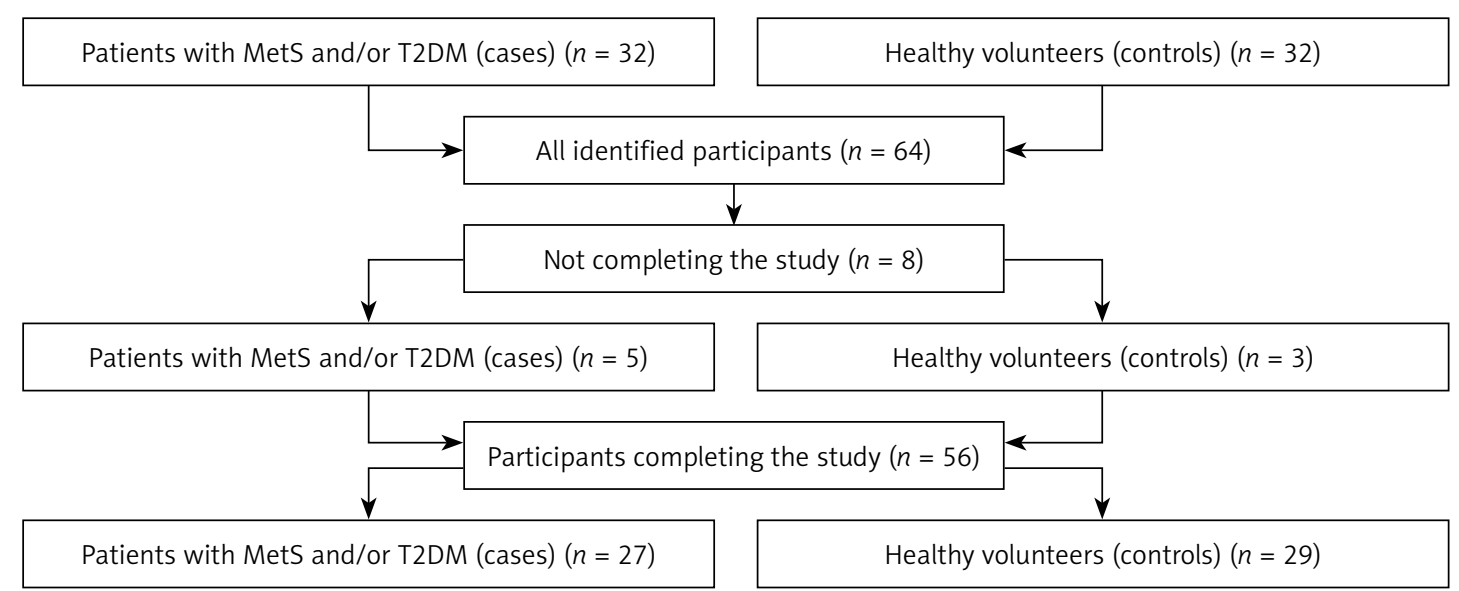

Figure 1. CONSORT-like flow-chart of study design, enrolment and analysis

A

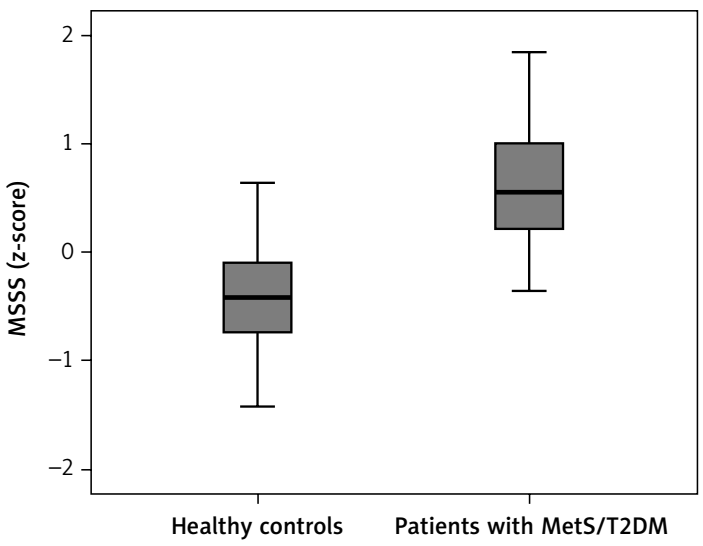

B

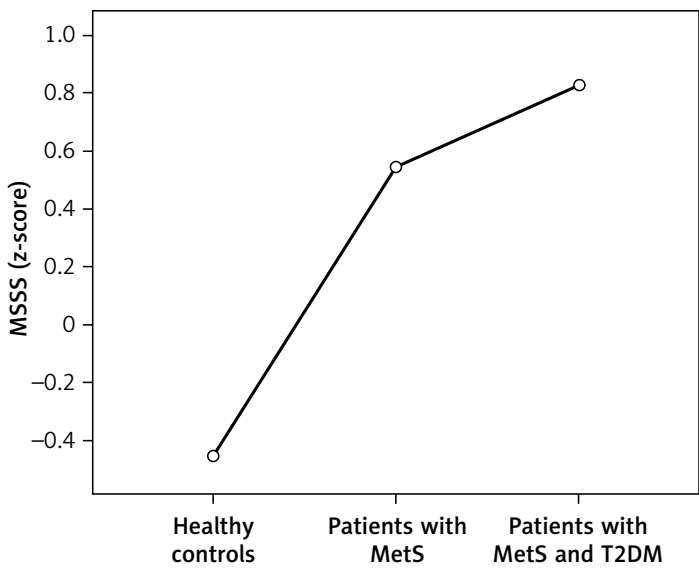

Figure 2. Metabolic syndrome severity score (MSSS) comparisons between healthy controls and patients with MetS/T2DM. A - Box-plots, illustrating the differences of MESS (z-score) between healthy controls and patients. Median, interquartile range (IQR) and the end-point values within 1.5 times IQR are indicated by the middle horizontal line, lower and upper box lines and whiskers, respectively. B - Results from ANOVA of the comparison between MSSS (z-score) in the 3 subgroups: healthy controls, patients with MetS alone and patients with both MetS and T2DM

The above findings, in terms of a possible relationship between MSSS and MetS/T2DM (outcome) as a further, second validating characteristic for this newly developed rule, were assessed by a logistic regression model. The model indicated a very strong and significant positive relationship (Figure $3 \mathrm{~A}$ ) of increasing probability of the outcome with increasing values of the MSSS $z$-score ( $p<0.001$, correct classification $>85 \%$ ); when the relationship was adjusted for gender, interesting differing patterns of this relationship were observed for males and females, although gender was not found to be significant as a covariate ( $p=$ 0.272) (Figure $3 \mathrm{~B}$ ).

Further confirmation of the above finding was also the association of each individual risk factor and MetS component by the odds ratio (OR) and, where this was not possible, by the $\chi^{2}$ or Fisher's exact test only (Table II). Clearly, the CVD risk factors are more frequent and with higher, more risky levels in the cases with MetS/T2DM than in the healthy controls.

\section{Associations of MSSS with CVD risk factors}

The establishment of higher MSSS values in Bulgarian patients with MetS/T2DM, including the statistically significant differences from Bulgarian controls (i.e., among a population that is different from that in the USA where the MSSS was initially derived and all initial relationships were first described), is one of the main elements in the process of validation of the new score for severity of the metabolic syndrome in another country, i.e., Bulgaria. Since it is well known that MetS and its severity, or progression, are important CVD risk factors, the third way by which such a continuous score may be validated further is to confirm statistically significant correlations with MetS components which had not been used in 
A

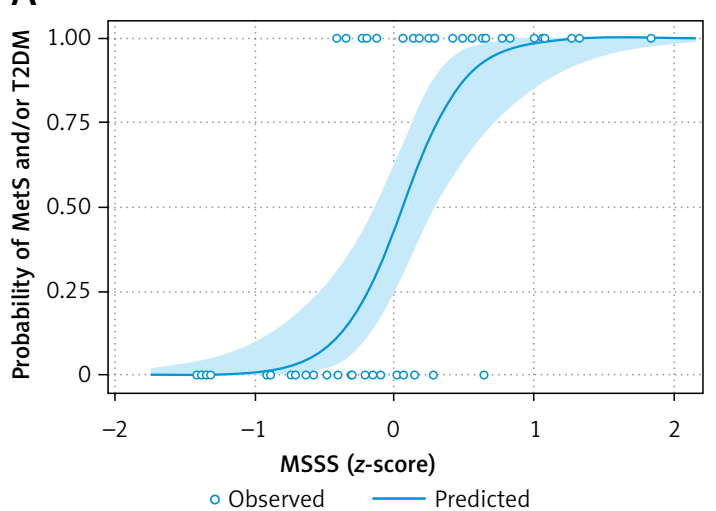

B

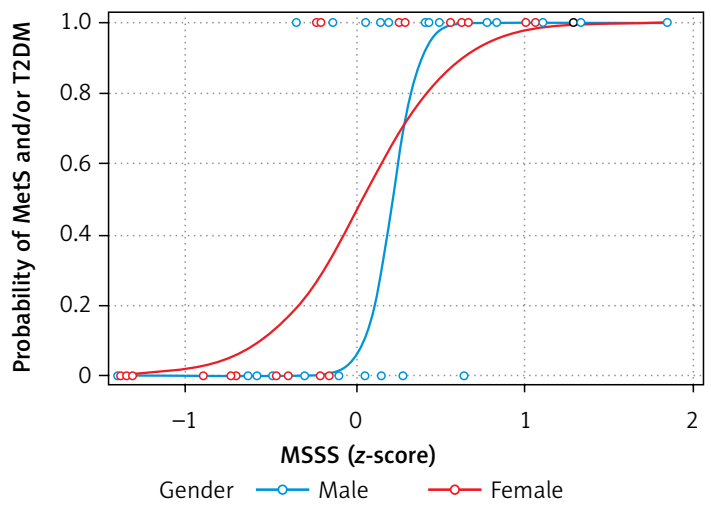

Figure 3. Logistic regression models of cases with MetS/T2DM and MSSS in all 56 participants. A - Probability of MetS/T2DM expressed as a nonlinear relationship along the MSSS ( $z$-score) range $(p<0.001)$. X-axis, MSSS (z-score); Y-axis, probability of MetS/T2DM (where $0.00=$ no MetS/T2DM; $1.00=$ MetS/T2DM) at cut-off probability of 0.50. B - Probability of MetS/T2DM expressed as a nonlinear relationship along the MSSS range, adjusted for gender $\left(p_{\text {mode }}<0.001\right)$. X-axis, MESS ( $z$-score); Y-axis, probability of MetS/T2DM (where $0.0=$ no MetS/T2DM; $1.0=$ MetS/T2DM) at cut-off probability of 0.5. Male, blue curve (steeper relationship); female, red curve (more flattened relationship); circles, individual values

Table II. Associations of socio-demographic, medical history and clinical characteristics with the presence of metabolic syndrome and/or type 2 diabetes mellitus

\begin{tabular}{|c|c|c|c|}
\hline Characteristics/risk factors & Categories & $\begin{array}{c}\text { Frequency } \\
\text { of MetS/T2DM in } \\
\text { each category (\%) }\end{array}$ & Odds ratio, OR $(p)^{*}$ \\
\hline Gender & Female/male & $37.8 / 68.4$ & $0.281(p=0.030)$ \\
\hline Education & Higher/secondary & $44.2 / 61.5$ & $0.495(p=0.273)$ \\
\hline Smoking & No/ex-smoker/yes & $45.8 / 50.0 / 50.0$ & $-(p=0.955)$ \\
\hline Medications & Yes/no & $81.8 / 33.3$ & $9.00(p=0.065)^{\#}$ \\
\hline Family history & Yes/no & $70.6 / 0.0$ & $-(p=0.021)^{\#}$ \\
\hline Family predisposition (parents) & Mother/father & $64.3 / 100$ & $-(p=0.515)^{\#}$ \\
\hline \multicolumn{4}{|l|}{ Diseases - main vascular risk factors: } \\
\hline Cardiovascular disease (CVD) & Yes/no & $65.2 / 35.5$ & $3.409(p=0.031)$ \\
\hline Dyslipidaemia (DLP) & Yes/no & $70.6 / 38.9$ & $3.771(p=0.031)$ \\
\hline Peripheral vascular disease (PVD) & Yes/no & $40.0 / 49.0$ & $0.694(p=0.99)^{\#}$ \\
\hline Hypertension (HT) & Yes/no & $100.0 / 26.3$ & $-(p=0.001)^{\#}$ \\
\hline \multicolumn{4}{|l|}{ Components of the metabolic syndrome ${ }^{* *}$ : } \\
\hline $\begin{array}{l}\mathrm{HDL}<1.00 \mathrm{mmol} / \mathrm{l} \text { (men) or } \\
\mathrm{HDL}<1.29 \mathrm{mmol} / \mathrm{l} \text { (women) or on treatment }\end{array}$ & Yes/no & $81.0 / 28.6$ & $10.625(p<0.001)^{\#}$ \\
\hline $\mathrm{TGL}>1.7 \mathrm{mmol} / \mathrm{l}$ or on treatment & Yes/no & $95.2 / 20.0$ & $80.000(p<0.001)^{\#}$ \\
\hline $\begin{array}{l}\text { Blood glucose }>5.6 \mathrm{mmol} / / \mathrm{l} \text { or } \\
\text { on treatment/T2DM }\end{array}$ & Yes/no & $65.5 / 29.6$ & $4.513(p<0.007)$ \\
\hline $\begin{array}{l}\mathrm{SBP}>130 \text { or } \mathrm{DBP}>85 \text { and/or } \\
\text { on anti-hypertensive therapy** }\end{array}$ & Yes/no & $94.1 / 28.2$ & $40.727(p<0.001)^{\#}$ \\
\hline $\begin{array}{l}\text { Waist circumference }>94 \mathrm{~cm} \text { (men) } \\
\text { or }>80 \mathrm{~cm} \text { (women) }\end{array}$ & Yes/no & $57.1 / 21.4$ & $4.889(p<0.030)^{\#}$ \\
\hline
\end{tabular}

$O R$, the statistical significance was determined by ${ }^{*} \chi^{2}$ test or "Fisher's exact test, was not calculated for the characteristics with null events or more than two categories; ${ }^{*}$ Defined according to the guidelines of the Bulgarian Institute for Metabolic Syndrome and International Diabetic Federation, MetS - metabolic syndrome, T2DM - type 2 diabetes mellitus, HDL - high-density lipoprotein, $T G L$ - triglycerides, SBP - systolic blood pressure, DBP - diastolic blood pressure. 


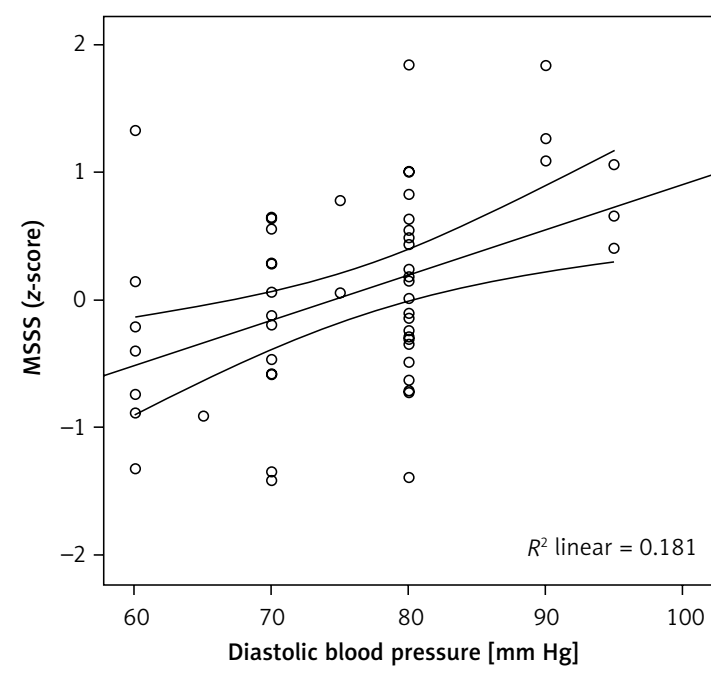

Figure 4. Linear relationship between diastolic blood pressure and MSSS. X-axis, diastolic blood pressure; Y-axis, MSSS (z-score); circles, individual values; diagonal line, best fitting regression model line; curves, $95 \%$ confidence intervals; $R^{2}$ is for illustrative purposes only (see text for more details)

the calculation of MSSS, other known CVD risk factors or validated CVD clinical prediction rules. In fact, as a third validating pattern, we revealed such positive correlations between MSSS and: (i) diastolic blood pressure $(\mathrm{DBP})-\mathrm{Rho}_{\text {Spearman }}=$ 0.399 ( $p<0.003$, Figure 4); (ii) predicted heart age (Rho $=0.368, p<0.007$ ); and (iii) QRISK2 score Rho $=0.524(p<0.001$, Figure 5$)$.

In this sense, as another albeit secondary confirmatory pattern, we can present the significant correlation between the predicted heart age and the calendar age $\left(\mathrm{Rho}_{\text {Spearman }}=0.381, p<0.005\right.$, not shown). We have to underline that the predicted

A

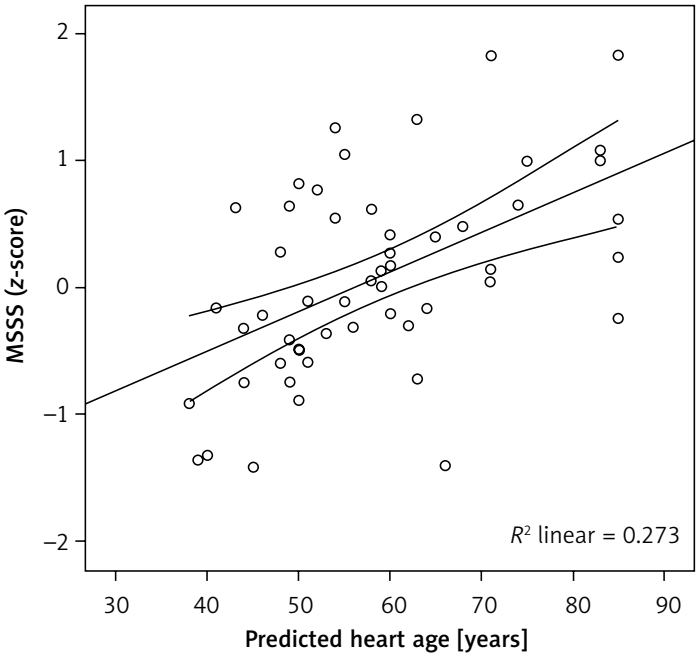

heart age (mean $=58.4)$ is higher by about 8.2 years $(p<0.001)$ than the mean calendar age of all participants (50.11, Table I) despite the fact that both cases and controls had the same mean calendar age.

\section{Discussion}

Our present work has applied the new MSSS, as developed in the USA, for the first time in Bulgaria. In their longitudinal study in 2015, Vishnu and co-authors reported a predictive accuracy of $80 \%$ for MSSS [14]. The calculated MSSS showed a positive correlation with the development of cardiovascular diseases or CVD-related outcomes and procedures (myocardial infarction, heart surgery, bypass, stroke) later in life [21]. Higher basic values were related to earlier age of CVD incidents (mean of 38 years) [26].

The clinical prediction rule, MSSS, by Gurka et al. [20] has been applied in our pilot study in Bulgaria and indicated significantly higher values in the 27 (48.2\%) patients with MetS/T2DM. It has proved to be feasible for application in a clinical setting for screening purposes and reliable in terms of consistency of the results: higher levels indicated higher probability of presence of MetS/T2DM (Figure 3 A), and there was a positive correlation of MSSS with both the predicted heart rate and QRISK score for 10-year cardiovascular risk.

In conclusion, we may consider that our pilot implementation in Bulgaria of the newly developed American score for MSSS is successful and has shown its feasibility, ease of use and consistency in terms of range and directions of the significant associations with MetS/T2DM and related CVD

B

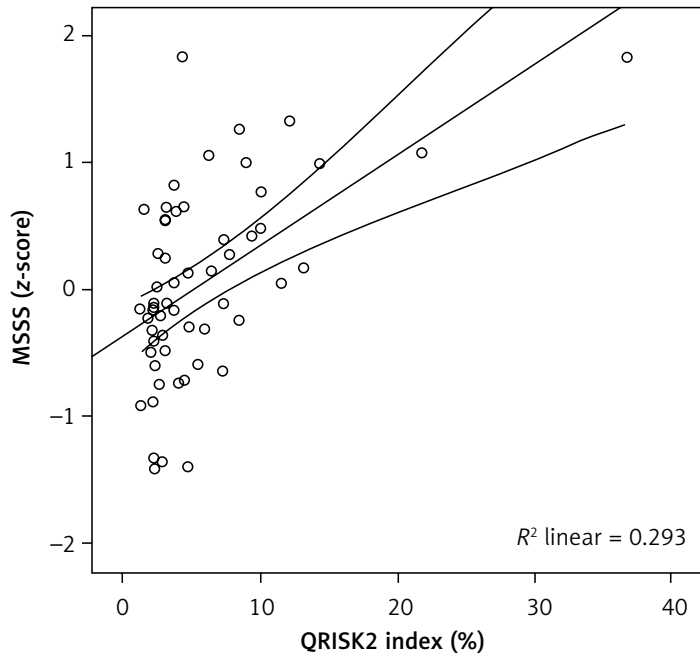

Figure 5. Linear relationship of MSSS with predicted heart age and QRISK2 index. A - X-axis, predicted heart age; $Y$-axis, MSSS (z-score); circles, individual values; diagonal line, best fitting regression model line; curves, $95 \%$ confidence intervals; $R^{2}$ is for illustrative purposes only (see text for more details); B - X-axis, QRISK2 index (\%); Y-axis, MSSS (z-score); circles, individual values; diagonal line, best fitting regression model line; curves, $95 \%$ confidence intervals; $R^{2}$ is for illustrative purposes only (see text for more details) 
risk factors. These results are original for Bulgaria and also presented the MSSS distribution among healthy volunteers, which may serve as the basis of further, large studies to establish normal, references standards for the Bulgarian population. The above findings may also be used for planning further trials for wider, prospective validation and impact analysis of MSSS in Bulgaria and, if necessary, for its further calibration, modification and updating.

\section{Conflict of interest}

The authors declare no conflict of interest.

\section{References}

1. Christov VI, Gocheva N, Petkova M, et al. A consensus of the Bulgarian Institute Metabolic Syndrome [Bulgarian]. Nauka Endocrinologia 2010; 2: 53-70.

2. Parvanova AI, Trevisan R, Iliev IP, et al. Insulin resistance and microalbuminuria: a cross-sectional, case-control study of 158 patients with type 2 diabetes and different degrees of urinary albumin excretion. Diabetes 2006; 55: 1456-62.

3. Parvanova A, Iliev I, Filipponi $M$, et al. Insulin resistance and proliferative retinopathy: a cross-sectional, case-control study in 115 patients with type 2 diabetes. J Clin Endocrinol Metab 2004; 89: 4371-6.

4. Mottillo S, Filion KB, Genest J, et al. The metabolic syndrome and cardiovascular risk: a systematic review and meta-analysis. J Am Coll Cardiol 2010; 56: 1113-32.

5. Hanley AJ. Prediction of type 2 diabetes mellitus with alternative definitions of the metabolic syndrome: the Insulin Resistance Atherosclerosis Study. Circulation 2005; 112: 3713-21.

6. Gami AS, Witt BJ, Howard DE, et al. Metabolic syndrome and risk of incident cardiovascular events and death. A systematic review and meta-analysis of longitudinal studies. J Am Coll Cardiol 2007; 49: 403-14.

7. Stefanov TS, Temelkova-Kurktschiev TS. The metabolic syndrome in Bulgaria. Folia Med (Plovdiv) 2011; 53: 5-14.

8. Borissova AM, Kovatcheva R, Shinkov A, et al. Prevalence and features of the metabolic syndrome in unselected Bulgarian population [Bulgarian]. Endocrinologia 2007; 12: 68-77.

9. Alberti KG, Zimmet P, Shaw J; IDF Epidemiology Task Force Consensus Group. The metabolic syndrome a new worldwide definition. Lancet 2005; 366: 1059-62.

10. Turker $Y$, Baltaci D, Turker $Y$, et al. Investigation of relationship of visceral body fat and inflammatory markers with metabolic syndrome and its components among apparently healthy individuals. Int J Clin Exp Med 2015; 8: 13067-77.

11. Atanassova PA, Angelova E, Tzvetanov P, Semerdjieva $M$, Dimitrov BD. Modelling of increased homocysteine in ischaemic stroke: post-hoc cross-sectional matched case-control analysis in young patients. Arq Neuropsiquiatr 2007; 65: 24-31.

12. Atanassova PA, Chalakova NT, Dimitrov BD. Diastolic blood pressure cut-off predicts major cerebrovascular events after minor ischaemic stroke: a post-hoc modelling study. Cent Eur J Med 2008; 3: 430-7.

13. Atanassova PA, Terzieva DD, Dimitrov BD. Impaired nocturnal melatonin in acute phase of ischaemic stroke: cross-sectional matched case-control analysis. J Neuroendocrinol 2009; 21: 657-63.

14. Vishnu A, Gurka MJ, DeBoer MD. The severity of the metabolic syndrome increases over time within individuals, independent of baseline metabolic syndrome status and medication use: the atherosclerosis rsk in communities study. Atherosclerosis 2015; 243: 278-85.

15. Kahn R, Buse J, Ferrannini E, Stern M. The metabolic syndrome: time for a critical appraisal: joint statement from the American Diabetes Association and the European Association for the Study of Diabetes. Diabetes Care 2005; 28: 2289-304.

16. Wijndaele K, Beunen G, Duvigneaud N, et al. A continuous metabolic syndrome risk score: utility for epidemiological analyses. Diabetes Care 2006; 29: 2329.

17. Viitasalo A, Lakka TA, Laaksonen DE, et al. Validation of metabolic syndrome score by confirmatory factor analysis in children and adults and prediction of cardiometabolic outcomes in adults. Diabetologia 2014; 57: 940-9.

18. Okosun IS, Lyn R, Davis-Smith M, Eriksen M, Seale P. Validity of a continuous metabolic risk score as an index for modeling metabolic syndrome in adolescents. Ann Epidemiol 2010; 20: 843-51.

19. Shafiee G, Kelishadi R, Heshmat R, et al. First report on the validity of a continuous metabolic syndrome score as an indicator for metabolic syndrome in a national sample of paediatric population - the CASPIAN-III study. Endokrynol Pol 2013; 64: 278-84.

20. Gurka MJ, Lilly CL, Oliver MN, DeBoer MD. An examination of sex and racial/ethnic differences in the metabolic syndrome among adults: a confirmatory factor analysis and a resulting continuous severity score. Metabolism 201; 63: 218-25.

21. DeBoer MD, Gurka MJ, Woo JG, Morrison JA. Severity of metabolic syndrome as a predictor of cardiovascular disease between childhood and adulthood: the Princeton Lipid Research Cohort Study. J Am Coll Cardiol 2015; 66: 755-7.

22. Hillier TA, Rousseau A, Lange C, et al. Practical way to assess metabolic syndrome using a continuous score obtained from principal components analysis. Diabetologia 2006; 49: 1528-35.

23. Raitakari OT, Porkka KV, Räsänen L, Rönnemaa T, Viikari JS. Clustering and six year cluster-tracking of serum total cholesterol, HDL-cholesterol and diastolic blood pressure in children and young adults. The Cardiovascular Risk in Young Finns Study. J Clin Epidemiol 199; 47: 1085-93.

24. Hippisley-Cox J, Coupland C, Vinogradova Y, et al. Predicting cardiovascular risk in England and Wales: prospective derivation and validation of QRISK2. BMJ 2008; 336: 1475-82.

25. D’Agostino RB, Vasan RS, Pencina MJ, et al. General cardiovascular risk profile for use in primary care: the Framingham Heart Study. Circulation 2008; 117: 743-53.

26. DeBoer MD, Gurka MJ, Woo JG, Morrison JA. Severity of the metabolic syndrome as a predictor of type 2 diabetes between childhood and adulthood: the Princeton Lipid Research Cohort Study. Diabetologia 2015; 58: 2745-52. 